\title{
The Role of Tongue Revascularization in Oncologic Resection and Reconstruction
}

\author{
Kenneth B. Lewoczko, MD ${ }^{1}$ \\ ${ }^{1}$ Division of Otolaryngology, University of Wisconsin, Madison, \\ Wisconsin
}

J Reconstr Microsurg Open 2016;1:56-58.

\author{
Aaron M. Wieland, MD ${ }^{1}$
}

Address for correspondence Kenneth B. Lewoczko, MD, Division of Otolaryngology, University of Wisconsin, K4/7 Clinical Science Center, 600 Highland Avenue, Madison, WI 53792

(e-mail: klewoczko@uwhealth.org).

Simultaneous sacrifice of both lingual arteries usually leads to critical compromise of the tongue's vascular supply and ischemic necrosis. ${ }^{1}$ As a result, simultaneous sacrifice of both lingual arteries has almost uniformly necessitated a total glossectomy. ${ }^{2}$ Although the bilateral facial arteries provide a minor vascular contribution to the tongue, situations where tongue remnants are preserved after bilateral lingual artery sacrifice usually result in eventual partial or total necrosis and sloughing of the tongue remnant. In scenarios where useful tongue tissue remains, revascularization of the devitalized remnant should be considered. The purpose of this article is review the available literature in this regard and to report a case of bilateral lingual artery sacrifice due to tumor involvement, with successful lingual artery interposition graft revascularization of the tongue.

\section{Case Presentation}

A 74-year-old gentleman was referred to our clinic with a history of a T4a N2c squamous cell carcinoma of the larynx with extension to the base of tongue, initially diagnosed 8 months previously and treated with primary concurrent chemoradiation. A positron emission topography (PET) scan done 4 months following completion of treatment revealed a hypermetabolic mass in the midline centered at the junction of the supraglottis and the base of tongue, suggestive of persistent disease (-Fig. 1), as well as an enlarged hypermetabolic left neck level II lymph node. A biopsy of the tongue base revealed moderately differentiated squamous cell carcinoma. The patient was presented at our multidisciplinary tumor board, and a recommendation was made for salvage surgery to include total laryngectomy with tongue base resection, bilateral neck dissections, and reconstruction with a pectoralis major myocutaneous flap.

Intraoperatively, the tumor encased both lingual arteries and hypoglossal nerves, necessitating sacrifice to achieve an oncologically sound resection. Bilateral selective neck dis-

received

May 26, 2015

accepted after revision

October 12, 2015

published online

January 21, 2016 sections (levels II-IV) were performed with preservation of the facial arteries to retain any facial artery contribution to the tongue through the floor of mouth. Even so, the remaining oral tongue was pale, and there was no bleeding when the tip was incised. In an effort to preserve the remaining oral tongue, an interposition arterial graft was performed. The proximal portion of the right lingual artery was dissected to its takeoff from the external carotid artery and harvested, yielding a $5-\mathrm{cm}$ arterial segment. The proximal and distal ends of the left lingual artery were identified and the segment of the right lingual artery was then used as an interposition graft (-Fig. 2). Upon completion of the microvascular anastomosis, there was normalization in the color of the tongue and bleeding from the cut made at the tip. A pectoralis major myocutaneous flap was then used to reconstruct the pharynx.

Six months postoperatively, the patient's tongue remained well vascularized (-Fig. 3); although he had no mobility of the tongue, his tactile sensation and taste were intact. He was consuming $\sim 50 \%$ of his calories by mouth, with the remainder coming through a gastrostomy tube.

\section{Discussion}

The vascular supply to the tongue is almost exclusively constituted by the lingual arteries and it is well established that at least one artery must be preserved to maintain tongue viability. ${ }^{1}$ Deep to the hyoglossus muscle, the lingual artery divides, providing dorsal lingual branches to the tongue base and two terminal branches anteriorly - the sublingual artery and the deep lingual artery ( - Fig. 4). The deep lingual artery supplies the anterior two-thirds of the tongue. This relationship is of particular importance in tongue base resection. The dorsal branch is often sacrificed, but if the lingual artery's origin is preserved, the deep lingual artery will continue to supply the anterior two-thirds of the tongue. The submental branch of the facial artery supplies the floor of mouth and provides a minor contribution to the body of the tongue. ${ }^{3}$
Copyright $\odot 2016$ by Thieme Medical Publishers, Inc., 333 Seventh Avenue, New York, NY 10001, USA. Tel: +1(212) 584-4662.

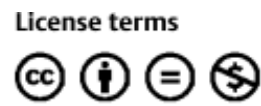

$10.1055 / \mathrm{s}-0035-1570507$ ISSN 2377-0813. 


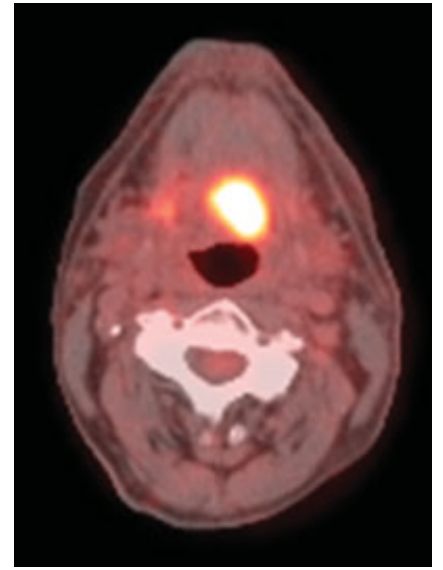

Fig. 1 Preoperative PET scan, showing a hypermetabolic lesion of the tongue base.

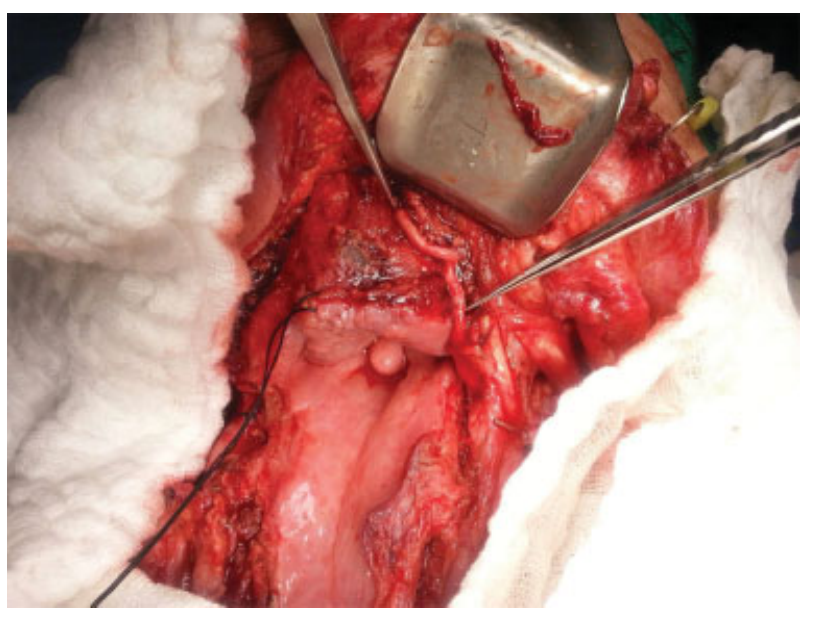

Fig. 2 The lingual artery interposition graft.

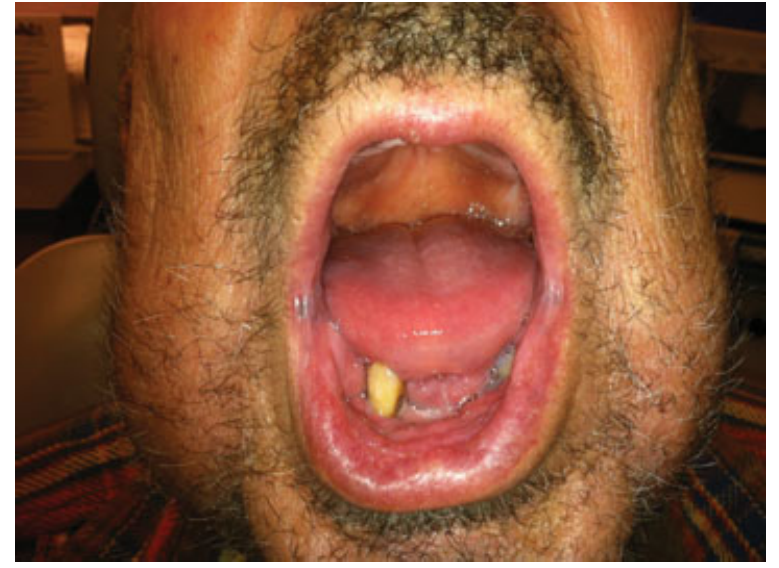

Fig. 3 The tongue 6 months postoperatively.

With rare exception, simultaneous sacrifice of the bilateral lingual arteries necessitates a total glossectomy. ${ }^{2}$ There have, however, been reports of rare exceptions to this rule, with one case of tongue survival after bilateral lingual artery ligation, ${ }^{1}$ and another report of the viability of a tongue set-back flap after lingual artery ligation. ${ }^{3}$ This is presumably due to adequate vascular contribution from branches of the facial artery. In our case, the facial artery was preserved bilaterally, and prior to revascularization, the tongue was pale and did not bleed when incised.

There also have been infrequent cases reported in which microvascular techniques were employed to preserve lingual tissue when the native vasculature was inadequate. Evans and colleagues reported a case in which the bilateral lingual arteries were sacrificed due to a deeply invading squamous cell carcinoma. An 8-cm radial forearm myocutaneous flap was used for reconstruction, with dissection of an additional $10 \mathrm{~cm}$ of the proximal radial artery. An end-to-side anastomosis was then performed between the proximal end of the

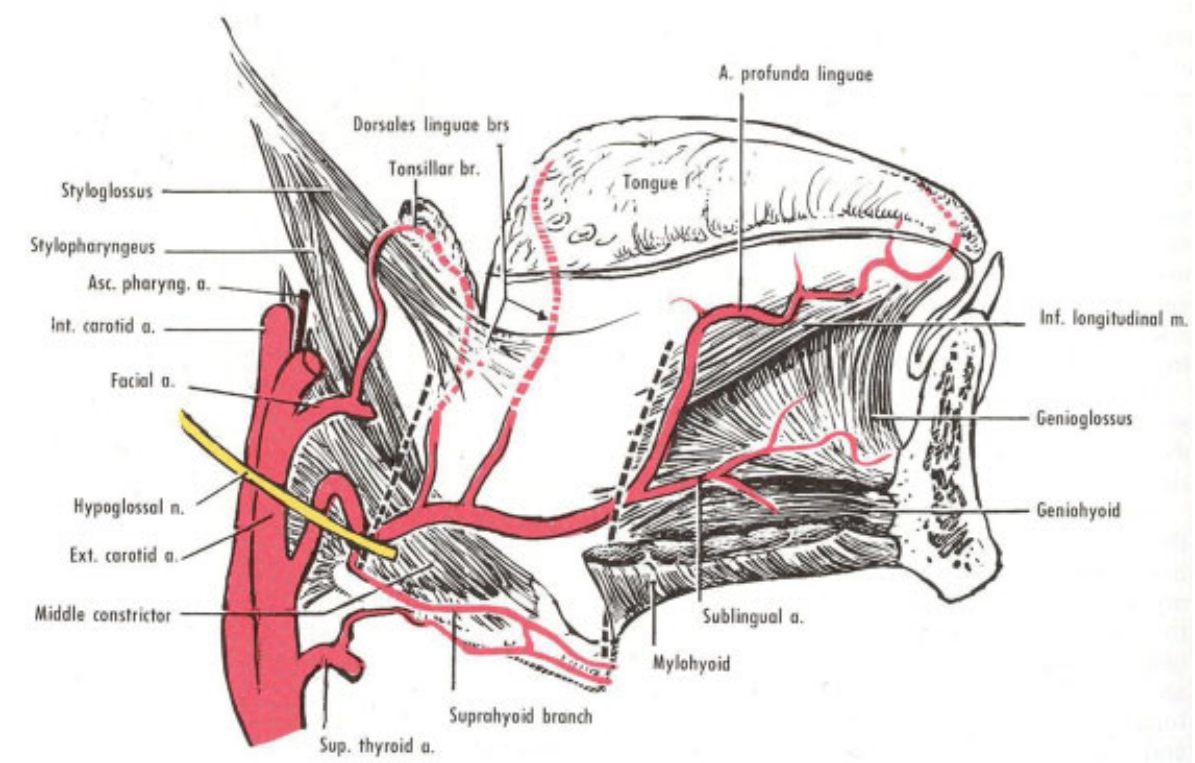

Fig. 4 The lingual artery. (Reprinted with permission from O'Rahilly R, Muller F, Carpenter S, Swenson R. The submandibular region. In: Basic Human Anatomy: A Regional Study of Human Structure. Hanover, NH: Darmouth Medical School; 2008:Fig. 49-4.) 
radial artery and the left external carotid artery (the right external carotid was sacrificed in the course of the neck dissection). The distal end of the radial artery was then anastomosed to the distal right lingual artery achieving revascularization with a flow-through configuration. ${ }^{4}$ The rectus myocutaneous flap has also been described with a flow-through design to revascularize the tongue. The inferior and superior epigastric arteries were used as the in-flow and out-flow, respectively. The inferior epigastric artery was anastomosed to the right superior thyroid artery, and the superior epigastric artery was anastomosed to the distal left lingual artery. ${ }^{5}$ The venous drainage of the tongue is more diffuse, and in both of these cases, there were no signs of venous congestion.

In addition to free tissue transfer, direct revascularization of the tongue has been reported using the facial artery. In that instance, the right facial artery was mobilized and anastomosed to the distal stump of the right lingual artery. Similar to our case, there was no evidence of any concerning venous congestion. ${ }^{6}$

In our case, native lingual artery was utilized for an interposition graft, which appears to be the first reported use of this technique. We were fortunate to have a sufficiently long proximal lingual artery to use as an interposition graft. If this had not been available, a variety of other vessels, both arterial and venous, could have been harvested for use as an interposition graft. In conclusion, with appropriate patient selection and favorable anatomy, lingual revascularization should be considered in cases necessitating bilateral lingual artery sacrifice.

\section{References}

1 Foster PK, Weed DT. Tongue viability after bilateral lingual artery ligation and surgery for recurrent tongue-base cancer. Ear Nose Throat J 2003;82(9):720-722, 724

2 Van Abel KM, Moore EJ. Surgical management of the base of tongue. Oper Tech Otolaryngol 2013;24:74-85

3 Lu TT, Zhang WF, Zhao YF. Reconstruction following resection of tongue base cancer using set-back tongue flap. Asian J Oral Maxillofac Surg 2004;16:218-223

4 Evans DM, Chevretton EB, Cole RP, Pereira JA, Morrison GA. Through-flow revascularization of the tongue using a radial forearm free flap. Br J Plast Surg 1994;47(6):419-421

5 Nakatsuka T, Harii K, Ebihara S. An inferior rectus abdominis bridge flap for revascularization of an ischemic tongue. Ann Plast Surg 1994;32(1):97-100

6 Shemen L, Romita M, Godfrey P, Godfrey N, Hamburg R. Microvascular revascularization of the tongue. J Reconstr Microsurg 1988; $4(4): 267-269$ 